\title{
Quantization of Acoustic Modes in Dumbbell Nanoparticles
}

\author{
Zuyuan Wang $\odot,{ }^{1,6, *}$ Hojin Kim $\odot,{ }^{2}$ Maria Secchi, ${ }^{3}$ Maurizio Montagna $\odot,{ }^{4}$ Eric M. Furst $\odot,{ }^{2}$ \\ Bahram Djafari-Rouhani $\odot^{5}$, and George Fytas $\oplus^{1, \dagger}$ \\ ${ }^{1}$ Max Planck Institute for Polymer Research, Ackermannweg 10, Mainz 55128, Germany \\ ${ }^{2}$ Department of Chemical \& Biomolecular Engineering, Allan P. Colburn Laboratory, University of Delaware, \\ Newark, Delaware 19716, USA \\ ${ }^{3}$ Department of Industrial Engineering, University of Trento, via Sommarive 9, I-38123 Trento, Italy \\ ${ }^{4}$ Dipartimento di Fisica, Universitá di Trento, via Sommarive 14, I-38123 Trento, Italy \\ ${ }^{5}$ Institut d'Électronique, de Microélectronique et de Nanotechnologie (IEMN), UMRCNRS 8520, Department of Physics, \\ University of Lille, Villeneuve d'Ascq 59655, France \\ ${ }^{6}$ Institute for Measurement and Automation, Division of Sensor Technology and Measurement Systems, \\ Bundeswehr University Munich, Werner Heisenberg Weg 39, 85579 Neubiberg, Germany
}

(Received 12 August 2021; accepted 5 January 2022; published 26 January 2022)

\begin{abstract}
The vibrational eigenmodes of dumbbell-shaped polystyrene nanoparticles are recorded by Brillouin light spectroscopy (BLS), and the full experimental spectra are calculated theoretically. Different from spheres with a degeneracy of $(2 l+1)$, with $l$ being the angular momentum quantum number, the eigenmodes of dumbbells are either singly or doubly degenerate owing to their axial symmetry. The BLS spectrum reveals a new, low-frequency peak, which is attributed to the out-of-phase vibration of the two lobes of the dumbbell. The quantization of acoustic modes in these molecule-shaped dumbbell particles evolves from the primary colloidal spheres as the separation between the two lobes increases.
\end{abstract}

DOI: 10.1103/PhysRevLett.128.048003

Spatially confined objects have quantized acoustic modes with signature frequencies that depend on the shape, size, elastic properties, and boundary conditions of the object [1-7]. Understanding the modes of particles with complex physical, chemical, and geometric parameters not only facilitates the development of acoustic quantization theories, but also provides a unique access to the elastic properties of small objects inaccessible by conventional methods $[8,9]$ and allows exploring resonant cavities in phononic materials [10]. For objects with dimensions in the submicrometer length scales, the frequencies of the acoustic resonance modes fall in the gigahertz $(\mathrm{GHz})$ range $[3,9,11-16]$ and can be effectively studied by Brillouin light spectroscopy (BLS).

So far, BLS studies have focused mainly on spheres $[12,14,17-19]$ due to the availability of theory, the challenge of synthesizing uniform, nonspherical particles, and the degeneracy of the resonance modes at a given angular momentum quantum number $l$. In recent extension to anisotropic shapes, the BLS spectra of ellipsoidal colloids are observed to be more complex due to the lifting of the

Published by the American Physical Society under the terms of the Creative Commons Attribution 4.0 International license. Further distribution of this work must maintain attribution to the author(s) and the published article's title, journal citation, and DOI. Open access publication funded by the Max Planck Society. degeneracy, $m=2 l+1[6,16]$. Yet, it is still barely understood how the reduced symmetry affects the eigenmodes of nanoparticles (NPs). In addition, interactions between spherical NPs (SPs) have been demonstrated to induce two prominent features: (1) the appearance of a contactinduced low-frequency mode $(n=1, l=1)$, and (2) the blueshift and peak splitting of the fundamental quadrupolar $(n=1, l=2)$ mode of SPs $[9,18,20,21]$. For plasmonic nanoparticles embedded in a polymer matrix, the optomechanically enhanced BLS spectra reveal the quadrupolar $(1,2)$ mode and an additional rattling mode, due to the coupling of the acoustic vibrations of the two spheres forming a dimer in close proximity [21-23]. However, the proximity in these studies is ill-defined, and the theoretical account of the vibration spectrum is only semiquantitative.

Dumbbell-shaped NPs (DBs) and their assemblies have been of great interest due to the anisotropic lattice structures, which can potentially provide direction-dependent optical and phononic properties [6,24]. The recent development of methods for synthesizing highly monodisperse DBs [25-27] has created opportunities to explore the shape dependence of vibrational modes. While the diameter is an exclusive parameter for a SP, a DB has three independent parameters: the diameters of the two lobes, $D_{1}$ and $D_{2}$, and the center-to-center distance between the lobes $d_{12}$. DBs offer a versatile platform for investigating the effect of particle shape symmetry on the eigenfrequencies and BLS vibration spectra, bridging colloidal atoms (spheres) and molecules (dumbbells). As $d_{12}$ increases, the shape of a DB 
changes from SP to DB and finally to two independent spheres with mechanical contact. The well-controlled proximity $d_{12}$ is an additional advantage compared to the metallic nanocomposites, and the use of submicron particles enables a strong BLS signal [15].

In this work, we measure the BLS spectrum of polystyrene DBs using BLS and compare their vibrational eigenmodes with those of SPs. The incident light (a Nd:YAG laser with a wavelength of $532 \mathrm{~nm}$ ) is directed to the SP and DB sample films to induce photon-phonon interactions, and the scattered light is collected by an aperture and focused by lenses to enter a tandem Fabry-Perot interferometer (JRS Scientific Instruments), with the light transmitted through the interferometer recorded by an avalanche photo diode. As the phonon wave vector $\mathbf{q}$ is ill-defined for opaque samples due to the multiple light scattering, the BLS spectrum reveals the resonance modes of the particles that are uniquely defined by the particles' geometric and elastic characteristics. We also develop a numerical method for calculating BLS spectra by combining the finite element method (FEM) and the theory of BLS [see Supplemental Material (SM), Sec. S2 for details [28] ]. The calculation results reproduce accurately the measured BLS spectra of SPs and DBs, and the vibrational patterns of the DB modes are interpreted in terms of the vibrational modes of the two lobes. A systematic tuning of $d_{12}$ provides an understanding of how near-frequency modes of the two composing spheres combine into a DB mode. A new low-frequency, $d_{12}$-sensitive peak is revealed and attributed to the out-ofphase rigid translations and rotations of the two lobes.

The vibrational modes of a free SP were first investigated by Lamb in 1881 [1] and later revisited and extended in 1982 [34]. They consist of two types: (1) spheroidal, $S(n, l, m)$, with $n \geq 1, l \geq 0$, and $-l \leq m \leq l$, and (2) torsional, $T(n, l, m)$, with $n \geq 1, l \geq 1$, and $-l \leq m \leq l$, where $n, l$, and $m$ are the quantization numbers analogous to the atomic orbitals. For SPs, the quantization number $m$ is often dropped, because the modes with the same $n$ and $l$ but different $m$ are degenerate, although their displacement patterns differ. The $S(1,1)$ and $T(1,1)$ modes of individual SPs, both threefold degenerate, represent the particle translation and rotation at zero frequency, respectively. For mechanically interacting SPs, however, both $S(1,1)$ and $T(1,1)$ have nonzero frequencies, and provide information on the interaction strength and contact area $[9,35]$. The eigenfrequencies and displacement fields of the $S$ and $T$ modes of SPs can be calculated $[34,36]$ as presented in SM, Sec. S1 [28]. The sequence of the eigenfrequencies is determined by $c_{L} / c_{T}$, while their absolute values depend on the longitudinal $c_{L}$ (or transverse $c_{T}$ ) sound velocities in the NP and the NP diameter $D$. In SM Table S1 [28], we show the lowest ten eigenfrequencies of a SP with $D=190 \mathrm{~nm}$, $c_{L}=2300 \mathrm{~m} / \mathrm{s}$, and $c_{T}=1210 \mathrm{~m} / \mathrm{s}$. For DBs, the eigenfrequencies and displacement fields are solved numerically. Besides the eigenfrequencies and eigenvectors of the
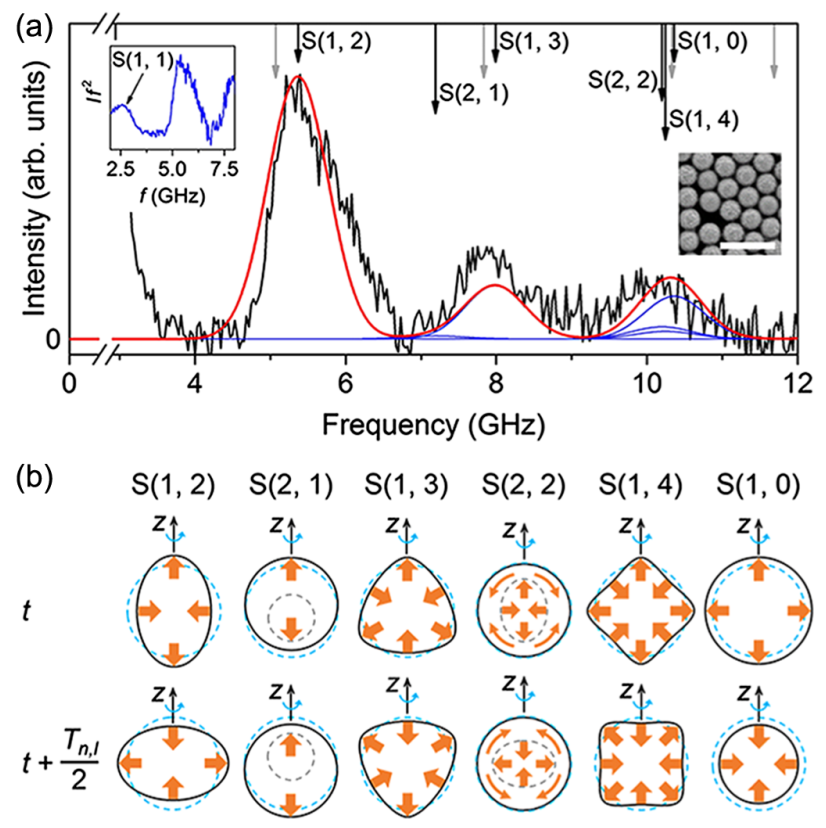

FIG. 1. Vibrational eigenmodes of polystyrene spherical nanoparticles. (a) Experimental (black line) and calculated (red line) BLS spectra of SP190. The blue lines represent the calculated intensities of the individual eigenmodes broadened to Gaussian profiles by the instrumental width $(0.35 \mathrm{GHz})$. Six spheroidal eigenmodes are indicated by black arrows. Four torsional eigenmodes are indicated by gray arrows. Left inset: a reduced spectrum, $I f^{2}$. Right inset: a scanning electron microscopy (SEM) image of the nanoparticles (scale bar: $500 \mathrm{~nm}$ ). The relatively low signal-to-noise ratio is due to the short accumulation time. (b) Illustration of the mode patterns (cross-sectional view, for $m=0$ ) of the six spheroidal eigenmodes in (a). The cyan, dashed circles represent nanoparticles at rest. The black, solid lines represent the nanoparticles surface in the specific vibrational eigenmodes. The gray, dashed lines indicate displacements in internal volumes. The orange arrows indicate local deformations. $t$ and $\left(t+T_{n, l} / 2\right)$ represent two chosen time instants separated by half of the vibrational period $T_{n, l}$ of the $(n, l)$ mode.

vibrational modes, we calculate the BLS scattering intensities [37] of these modes to fully account for the BLS experiment (SM, Sec. S2 [28]).

We first investigate the vibrational eigenmodes of polystyrene SPs with $D=190 \pm 4 \mathrm{~nm}$ (SP190) [right inset to Fig. 1(a); SM, Sec. S3 and Fig. S1(a)]. The experimental BLS spectrum of SP190, obtained from a measurement in the transmission geometry (SM, Sec. S4), features three peaks in the range of $4-12 \mathrm{GHz}$ [Fig. 1(a)]. An additional, broad low-frequency Brillouin peak, centered at about $2.5 \mathrm{GHz}$, appears in the plot of the reduced intensity $I f^{2}$ [left inset to Fig. 1(a)]. This peak, which is absent in the spectrum of a free sphere, is designated as $S(1,1)$, as it stems from the zero-frequency rigid translations of the sphere and can be attributed to the propagation of longitudinal sound waves due to physical contact between adjacent spheres [9]. We further calculate the 
BLS spectrum [red line in Fig. 1(a)] to identify the contributions of the individual eigenmodes (SM, Sec. S2). The first ten eigenfrequencies (up to $12 \mathrm{GHz}$ ), including six spheroidal and four torsional modes, agree well with those in Table S1 computed using Lamb's theory [1]. The vibrational patterns of the spheroidal modes with $m=0$ at the six frequencies are shown in Fig. 1(b), whereas those of the four torsional modes are shown in Fig. S2. A complete list of the eigenfrequencies (up to $12 \mathrm{GHz}$ ) and their displacement fields are shown in Fig. S3.

The BLS intensities of the torsional modes are zero, as these modes cause no change in the NP volume or shape (i.e., no change in the polarizability of the particle). The nonzero BLS intensity of the spheroidal modes confirms that both even- and odd- $l$ eigenmodes contribute to BLS [15]. While the first peak is contributed solely by the $S(1,2)$ quadrupolar mode, the second peak is contributed by two modes, $S(2,1)$ and $S(1,3)$. The third peak includes contributions from three modes, $S(2,2), S(1,4)$, and $S(1,0)$, although the intensities of the first two modes are significantly lower than the third one. Figure 1(a) implies that a quantitative analysis of a BLS spectrum requires considering both the eigenfrequencies and intensities of the modes, because the peaks in the BLS spectrum may each include contributions from multiple eigenmodes $[9,12]$. Furthermore, the relative intensities of the higherfrequency peaks with respect to the first one increase, as $D / \lambda$ increases (Fig. S4) [15]. We note that the spherical particles possess high sphericity [Figs. S1(a) and S1(c)]. A slight asphericity and small irregularities on the particle surface have a minor effect on the vibrational eigenfrequencies and eigenmodes, as revealed by the FEM calculations (Figs. S5 and S6).

The eigenmodes of DBs are more complex due to their lower symmetry. The SEM image [right inset to Fig. 2(a); SM, Sec. S3 and Fig. S1(b)] is used to estimate the three geometric parameters, including $D_{1}=232 \pm 9 \mathrm{~nm}, D_{2}=$ $214 \pm 10 \mathrm{~nm}$, and $d_{12}=151 \pm 9 \mathrm{~nm}$. For these DBs, denoted as DB232-151-214, the recorded BLS spectrum [black line in Fig. 2(a)] reveals five peaks labeled as $A, B$, $C, D$, and $E$. We calculate the BLS spectrum of a single DB232-151-214 (SM, Sec. S2) with the same sound velocities of SP190. The reduced symmetry and the consequent $m$ splitting renders the number of observed peaks in the BLS spectrum [Fig. 2(a)] much larger than for the corresponding SP190 [Fig. 1(a)]. The characteristic eigenmodes under peaks $A-E$ are labeled, with the focus on peaks $A$ and $B$. Peak $A$ includes the contributions from three modes, denoted as $(A 1)_{2},(A 2)$, and $(A 3)_{2}$, which are doubly (indicated by the subscript " 2 "), singly, and doubly degenerate, respectively. Peak $B$ is contributed by eight modes, denoted as $(B 1)_{2}$ to $(B 8)_{2}$, while peaks $C-E$ include contributions from even more modes. The vibrational patterns of the five singly degenerate modes indicated as $(A 2),(B 6),(C 1),(D 1)$, and (E1) in Fig. 2(a) are

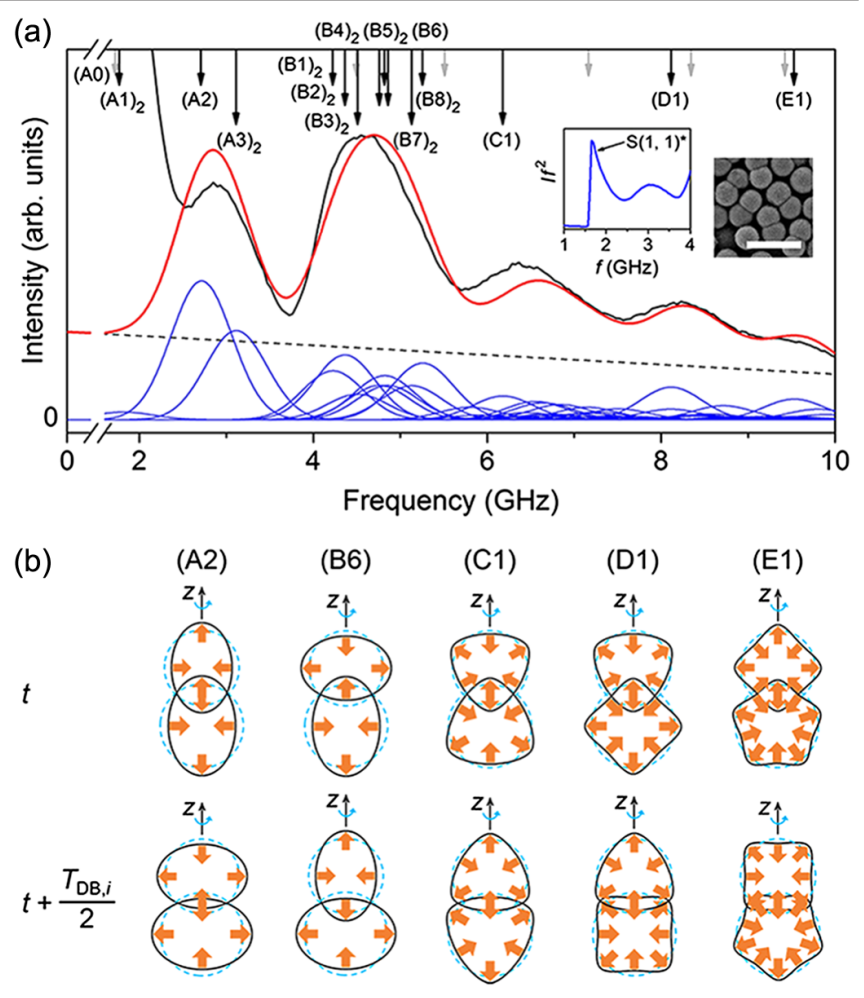

FIG. 2. Vibrational eigenmodes of polystyrene dumbbellshaped nanoparticles. (a) Experimental (black line) and theoretical (red line) BLS spectra of DB232-151-214. The blue lines represent the theoretical intensities of the individual eigenmodes broadened by the instrumental width $(0.35 \mathrm{GHz})$ and the dashed line denotes a linear background added to the calculated total spectrum to account for the Rayleigh wing in the experimental spectrum. Characteristic eigenmodes in the five peaks ( $A$ to $E$ ) are indicated by black arrows. The subscript " 2 " means the mode is doubly degenerate. Six pure torsional eigenmodes are indicated by gray arrows. Left inset: a reduced spectrum, $I f^{2}$ (the abrupt intensity drop at around $1.6 \mathrm{GHz}$ is due to the shutter). Right inset: an SEM image of the nanoparticles (scale bar: $500 \mathrm{~nm}$ ). (b) Illustration of the mode patterns (cross-sectional view) of the six singly degenerate spheroidal eigenmodes in (a). See the Fig. 1(b) caption for more details. $T_{\mathrm{DB}, i}$ represents the vibrational period of the corresponding eigenmode.

shown in Fig. 2(b), whereas those of the six singly degenerate modes indicated by gray arrows are shown in Fig. S7.

It is reasonable to envisage the vibrational patterns of the DBs as those of the two lobes, seen as spheres. For example, (A2) and (B6) have patterns that mimic the patterns of two $S(1,2)$ modes of the individual lobes vibrating in phase and out of phase [Fig. 2(b)], respectively. Modes of the individual lobes with different $l$ 's interact too, as seen in the vibrational patterns of $(D 1)$ and $(E 1)$. (D1) can be considered as a hybridization of the $S(1,3,0)$ mode of the top (smaller) lobe with the $S(1,4,0)$ of the bottom (larger) lobe [Fig. 2(b)]. (E1) can be considered as a hybridization of the $S(1,4,0)$ mode of the top (smaller) lobe with the $S(1,5,0)$ of bottom (larger) lobe [Fig. 2(b)]. 
This hybridization is favored by a small mismatch between the frequency of a mode with a smaller $l$ in the smaller sphere and that of a mode with a larger $l$ in the larger sphere. The similar patterns of the two sphere modes in the overlap region also facilitate the hybridization. We note that this schematic representation in terms of the sphere modes is not general. It works well only for DBs with high $d_{12}$ values, where the two spheres are well evident and interact via a disk with a small radius $r_{0}$ (i.e., $r_{0} \ll \min \left\{D_{1}, D_{2}\right\}$ ). Based on the displacement fields of the DB232-151-214 eigenmodes (Fig. S8), only a few modes resemble those of the two individual lobes. The resemblance occurs, when only one sphere vibrates, while the second sphere has no mode with a comparable frequency.

To understand the vibrational eigenmodes of DB232151-214, we calculate the eigenfrequencies of a series of DBs with varied $d_{12}$ [Fig. 3(a)]. At $d_{12} \leq 9 \mathrm{~nm}$, the two lobes of the DB overlap, and the eigenmodes of the "spherical" DB are similar to those of SP232. The corresponding eigenfrequencies of the torsional and spheroidal modes are indicated on the left $y$ axis in Fig. 3(a) by empty and filled triangles, respectively. As $d_{12}$ increases, the eigenfrequencies separate into $(2 l+1)$ branches due to the lifting of the degeneracy. Specifically, a $T(n, l)$ or $S(n, l)$ mode separates into one singly and $l$ doubly degenerate mode, which correspond to $m=0$ and $m= \pm 1, \pm 2, \ldots, \pm l$, respectively. In Fig. 3(a), the singly degenerate modes originating from $T(n, l, m=0)$ $[S(n, l, m=0)]$ are represented by dashed (solid), blue lines, whereas the remaining doubly degenerate modes are shown by solid, red lines. Among the DB eigenmodes, only those originating from $T(n, l, m=0)$ have zero Brillouin intensity, while the others are all Brillouin active. As $d_{12}$ approaches $\left(D_{1}+D_{2}\right) / 2=223 \mathrm{~nm}$, the eigenmodes become a combination of those of the two lobes (i.e., SP232 and SP214). Concomitantly, the frequencies of the lowest six eigenmodes, shown by the lowest four lines in Fig. 3(a), vanish. Among the six vanishing modes, the lower three and higher three modes can be assigned to the rotational and translational modes of the two spheres, respectively. We note that a single DB has three rotational and three translational modes, whereas two free SPs have six rotational and six translational modes. The six disappearing modes are the zero-frequency translations and rotations of the whole DB. Moreover, the DB eigenfrequencies collapse into those of SP232 and SP214, as indicated by the black and green triangles on the right $y$ axis in Fig. 3(a), respectively.

The modes contributing to peaks $A$ and $B$ in Fig. 2(a) are highlighted in Fig. 3(a). At large $d_{12}$, the mode (A2) is well represented by the nearly rigid translation of the two spheres along the $z$ axis in opposite directions [i.e., by the $S(1,1,0)$ modes of the individual lobes vibrating outof-phase]. As $d_{12}$ decreases, (A2) gains nature of the $S(1,2)$ modes of the two lobes. For even smaller $d_{12},(A 2)$ converts
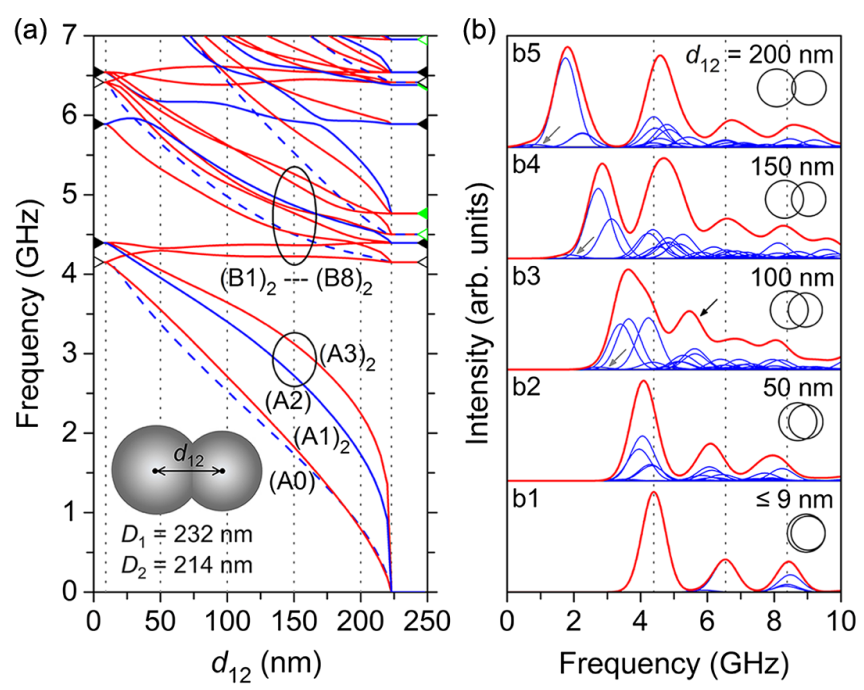

FIG. 3. Vibrational eigenmodes and BLS spectra of asymmetric dumbbell-shaped nanoparticles. (a) Eigenmodes of DB232$d_{12}$-214 at varying $d_{12}$ 's. The red lines denote doubly degenerate modes. The blue, solid (dashed) lines represent singly degenerate modes with nonzero (zero) Brillouin intensities. The eigenmodes contributing to peaks $A$ and $B$ in Fig. 2(a) are circled. The black, filled (open) triangles denote spheroidal (torsional) eigenmodes of SP232. From bottom to top: $T(1,2), S(1,2), S(2,1), T(1,3)$, and $S(1,3)$. The green, filled (open) triangles denote spheroidal (torsional) eigenmodes of SP214. From bottom to top: $T(1,2)$, $S(1,2), S(2,1)$, and $T(1,3)$. Inset: a schematic of the DB. (b) Calculated BLS spectra of DB232- $d_{12}-214$ at five $d_{12}$ 's. The gray arrows in (b3)-(b5) indicate the lowest-frequency mode. The black arrow in (b3) indicates the new, intermediate-frequency peak at $d_{12}=100 \mathrm{~nm}$. See the caption of Fig. 2(a) for more details. Insets: schematics of DBs.

to $S(1,2)$ of SP232. Similar arguments hold for the doubly degenerate $(A 3)_{2}$ modes, but in this case the $S(1,1,1)$ and $S(1,1,-1)$ modes (i.e., translations of the two spheres along the $x$ and $y$ directions) are present for high $d_{12}$ values. As $d_{12}$ decreases, $(A 3)_{2}$ gain nature of $S(1,2)$ of the two spheres, while for even smaller $d_{12}$, they convert to $S(1,2)$ of SP232. On the other hand, for high $d_{12}$ values, the $(A 0)$ and $(A 1)_{2}$ modes correspond to pure, out-of-phase rotations of the two spheres with respect to the $z$ axis and $x$ (or $y$ ) axis, respectively. They become three $T(1,1, m)$ modes of SP232 at very small $d_{12}$. For intermediate $d_{12}$, the $(A 1)_{2}$ modes are not purely torsional. They are hybridized with the $S(1,1)^{*}$ modes [left inset to Fig. 2(a)] and thereby gain some Brillouin activity that produces the weak, lowest-frequency mode [blue line indicated by a gray arrow in Figs. 3(b3)-3(b5)]. These examples demonstrate that a perturbative approach based on the knowledge of the modes of the two spheres helps to understand the nature of the DB vibrations. The eight modes contributing to peak $B$ originate from the $T(1,2), S(1,2), S(2,1), T(1,3)$, and $S(1,3)$ modes of SP232. Alternatively, they could be considered to result from the $T(1,2)$ and $S(1,2)$ modes 
of SP232 and SP214. We note that the (B6) mode is closely related to the $S(2,1)$ mode of SP232 at small $d_{12}$ and to the $S(1,2)$ of SP232 at large $d_{12}$. Therefore, as $d_{12}$ increases from $\left(D_{1}-D_{2}\right) / 2$ to $\left(D_{1}+D_{2}\right) / 2$, the nature of the DB eigenmodes changes.

To investigate how $d_{12}$ affects the vibration spectrum of DBs, we calculate the BLS spectra at five $d_{12}$ values [Fig. 3(b)]. For $d_{12} \leq 9 \mathrm{~nm}$, the dumbbell shape becomes spherical, and the BLS spectrum is similar to that of SP190 [Fig. 1(a)]; due to the different diameters, the eigenfrequencies are shifted $\left(f \sim D^{-1}\right)$ [13]. At $d_{12}=50 \mathrm{~nm}$, the NP has a prolate ellipsoidal shape, and the anticipated degeneracy lifting is observed. The total spectrum resembles that of SP232, except for a slight redshift. At $d_{12}=100 \mathrm{~nm}$, the spectrum becomes more complicated with a broadened first peak and the appearance of a new peak [indicated by the black arrow in Fig. 3(b3)] with a frequency between those of the first two peaks of SP232. As $d_{12}$ further increases, a strong, low-frequency peak appears, and it gradually becomes separated from the higher-frequency peaks. With increasing $d_{12}$, its frequency decreases, and its relative intensity increases. The three higher-frequency peaks in the DB spectrum resemble those of SP232 with slight modifications caused by the different diameters of the two DB lobes. We also conduct calculations on symmetric DBs with $D_{1}=D_{2}=200 \mathrm{~nm}$ and $d_{12}$ varying from 0 to $200 \mathrm{~nm}$. The peak splitting of DB200- $d_{12}-200$ (Fig. S9) is similar to that of DB232$d_{12}$-214 (Fig. 3). In the limit of $d_{12}=\left(D_{1}+D_{2}\right) / 2$, the new, low-frequency peak resembles the low-frequency, rattling mode in the BLS spectrum of symmetric metallic dimers with mechanical contact $[21,23]$.

In conclusion, we report the measured and calculated BLS spectra of DBs with well-defined proximity $d_{12}$. As $d_{12}$ increases, the spectrum evolves from that of a SP to that of a $\mathrm{DB}$, revealing a new, low-frequency peak that originates from the out-of-phase vibration of the two lobes. The BLS spectrum of a DB includes contributions from all spheroidal and torsional eigenmodes, except for those singly degenerate torsional modes that cause no change in the particle volume or shape. The calculation method, which combines the FEM and theory of BLS, and predicts BLS spectra in close agreement with experiments, could be extended to other particle shapes and thus advance the particle vibration spectroscopy. The elucidation of the vibration eigenmodes of DBs and the well-controlled tunability of their geometric parameters (i.e., $D_{1}, D_{2}$, $d_{12}$ ) are envisaged to promote the development of a wide range of DB-based phononic crystals with directiondependent, tunable band gaps, which could have potential applications in areas such as nanomaterials and on-chip devices by providing more degrees of freedom [38] in wave-based filtering and computing.
This work was financially supported by ERC AdG SmartPhon (Grant No. 694977). E. M. F. acknowledges that this material is based upon work supported by the National Science Foundation under Grant No. CBET1637991.

*Corresponding author.

wang.zuyuan@mpip-mainz.mpg.de

Corresponding author.

fytas@mpip-mainz.mpg.de

[1] H. Lamb, On the vibrations of an elastic sphere, Proc. London Math. Soc. s1-13, 189 (1881).

[2] L. Saviot, B. Champagnon, E. Duval, I. A. Kudriavtsev, and A.I. Ekimov, Size dependence of acoustic and optical vibrational modes of CdSe nanocrystals in glasses, J. Non-Cryst. Solids 197, 238 (1996).

[3] T. Still, R. Sainidou, M. Retsch, U. Jonas, P. Spahn, G. P. Hellmann, and G. Fytas, The "music" of core-shell spheres and hollow capsules: Influence of the architecture on the mechanical properties at the nanoscale, Nano Lett. 8, 3194 (2008).

[4] L. Saviot and D. B. Murray, Acoustic vibrations of anisotropic nanoparticles, Phys. Rev. B 79, 214101 (2009).

[5] D. Schneider, M. Schmitt, C. M. Hui, R. Sainidou, P. Rembert, K. Matyjaszewski, M. R. Bockstaller, and G. Fytas, Role of polymer graft architecture on the acoustic eigenmode formation in densely polymer-tethered colloidal particles, ACS Macro Lett. 3, 1059 (2014).

[6] P. J. Beltramo, D. Schneider, G. Fytas, and E. M. Furst, Anisotropic Hypersonic Phonon Propagation in Films of Aligned Ellipsoids, Phys. Rev. Lett. 113, 205503 (2014).

[7] T. Brunet, A. Merlin, B. Mascaro, K. Zimny, J. Leng, O. Poncelet, C. Aristégui, and O. Mondain-Monval, Soft 3D acoustic metamaterial with negative index, Nat. Mater. 14, 384 (2015).

[8] E. Kang, H. Kim, L. A. G. Gray, D. Christie, U. Jonas, B. Graczykowski, E. M. Furst, R. D. Priestley, and G. Fytas, Ultrathin shell layers dramatically influence polymer nanoparticle surface mobility, Macromolecules 51, 8522 (2018).

[9] H. Kim, Y. Cang, E. Kang, B. Graczykowski, M. Secchi, M. Montagna, R. D. Priestley, E. M. Furst, and G. Fytas, Direct observation of polymer surface mobility via nanoparticle vibrations, Nat. Commun. 9, 2918 (2018).

[10] Z. Liu, X. Zhang, Y. Mao, Y. Y. Zhu, Z. Yang, C. T. Chan, and P. Sheng, Locally resonant sonic materials, Science $\mathbf{2 8 9}$, 1734 (2000).

[11] R. S. Penciu, G. Fytas, E. N. Economou, W. Steffen, and S. N. Yannopoulos, Acoustic Excitations in Suspensions of Soft Colloids, Phys. Rev. Lett. 85, 4622 (2000).

[12] M. H. Kuok, H. S. Lim, S. C. Ng, N. N. Liu, and Z. K. Wang, Brillouin Study of the Quantization of Acoustic Modes in Nanospheres, Phys. Rev. Lett. 90, 255502 (2003).

[13] W. Cheng, J. J. Wang, U. Jonas, W. Steffen, G. Fytas, R. S. Penciu, and E. N. Economou, The spectrum of vibration modes in soft opals, J. Chem. Phys. 123, 121104 (2005).

[14] Y. Li, H. S. Lim, S. C. Ng, Z. K. Wang, M. H. Kuok, E. Vekris, V. Kitaev, F.C. Peiris, and G. A. Ozin, 
Micro-Brillouin scattering from a single isolated nanosphere, Appl. Phys. Lett. 88, 023112 (2006).

[15] T. Still, M. Mattarelli, D. Kiefer, G. Fytas, and M. Montagna, Eigenvibrations of submicrometer colloidal spheres, J. Phys. Chem. Lett. 1, 2440 (2010).

[16] D. Schneider, P. J. Beltramo, M. Mattarelli, P. Pfleiderer, J. Vermant, D. Crespy, M. Montagna, E. M. Furst, and G. Fytas, Elongated polystyrene spheres as resonant building blocks in anisotropic colloidal crystals, Soft Matter 9, 9129 (2013).

[17] T. Still, W. Cheng, M. Retsch, R. Sainidou, J. Wang, U. Jonas, N. Stefanou, and G. Fytas, Simultaneous Occurrence of Structure-Directed and Particle-Resonance-Induced Phononic Gaps in Colloidal Films, Phys. Rev. Lett. 100, 194301 (2008).

[18] M. Mattarelli, M. Montagna, T. Still, D. Schneider, and G. Fytas, Vibration spectroscopy of weakly interacting mesoscopic colloids, Soft Matter 8, 4235 (2012).

[19] M. He, J. P. Gales, É. Ducrot, Z. Gong, G.-R. Yi, S. Sacanna, and D. J. Pine, Colloidal diamond, Nature 585, 524 (2020).

[20] A. L. Tchebotareva, M. A. van Dijk, P. V. Ruijgrok, V. Fokkema, M. H. S. Hesselberth, M. Lippitz, and M. Orrit, Acoustic and optical modes of single dumbbells of gold nanoparticles, ChemPhysChem 10, 111 (2009).

[21] A. Girard, H. Gehan, A. Mermet, C. Bonnet, J. Lermé, A. Berthelot, E. Cottancin, A. Crut, and J. Margueritat, Acoustic mode hybridization in a single dimer of gold nanoparticles, Nano Lett. 18, 3800 (2018).

[22] A. Girard, H. Gehan, A. Crut, A. Mermet, L. Saviot, and J. Margueritat, Mechanical coupling in gold nanoparticles supermolecules revealed by plasmon-enhanced ultralow frequency Raman spectroscopy, Nano Lett. 16, 3843 (2016).

[23] A. Noual, E. Kang, T. Maji, M. Gkikas, B. Djafari-Rouhani, and G. Fytas, Optomechanic coupling in Ag polymer nanocomposite films, J. Phys. Chem. C 125, 14854 (2021).

[24] J. D. Forster, J.-G. Park, M. Mittal, H. Noh, C. F. Schreck, C. S. O'Hern, H. Cao, E. M. Furst, and E. R. Dufresne, Assembly of optical-scale dumbbells into dense photonic crystals, ACS Nano 5, 6695 (2011).

[25] J.-G. Park, J. D. Forster, and E. R. Dufresne, High-yield synthesis of monodisperse dumbbell-shaped polymer nanoparticles, J. Am. Chem. Soc. 132, 5960 (2010).

[26] E. B. Mock, H. De Bruyn, B. S. Hawkett, R. G. Gilbert, and C. F. Zukoski, Synthesis of anisotropic nanoparticles by seeded emulsion polymerization, Langmuir 22, 4037 (2006).

[27] E. B. Mock and C. F. Zukoski, Emulsion polymerization routes to chemically anisotropic particles, Langmuir 26, 13747 (2010).

[28] See Supplemental Material at http://link.aps.org/ supplemental/10.1103/PhysRevLett.128.048003 for details and additional results of the theoretical calculations, nanoparticle synthesis, and Brillouin light scattering experiments, which includes Refs. [29-33].

[29] A. C. Erigen and E. S. Suhubi, Elastodynamics (Academic Press, New York, 1975), Vol. 2.

[30] B. J. Berne and R. Pecora, Dynamic Light Scattering: With Applications to Chemistry, Biology, and Physics (Courier Dover Publications, Mineola, 2000).

[31] Y. Chonde and I. M. Krieger, Emulsion polymerization of styrene with ionic comonomer in the presence of methanol, J. Appl. Polym. Sci. 26, 1819 (1981).

[32] J. W. Goodwin, J. Hearn, C. C. Ho, and R. H. Ottewill, Studies on the preparation and characterisation of monodisperse polystyrene lattices, Colloid Polym. Sci. 252, 464 (1974).

[33] A. Crut, P. Maioli, N. Del Fatti, and F. Vallée, Acoustic vibrations of metal nano-objects: Time-domain investigations, Phys. Rep. 549, 1 (2015).

[34] A. Tamura, K. Higeta, and T. Ichinokawa, Lattice vibrations and specific heat of a small particle, J. Phys. C 15, 4975 (1982).

[35] V. Babacic, J. Varghese, E. Coy, E. Kang, M. Pochylski, J. Gapinski, G. Fytas, and B. Graczykowski, Mechanical reinforcement of polymer colloidal crystals by supercritical fluids, J. Colloid Interface Sci. 579, 786 (2020).

[36] E. Duval, Far-infrared and Raman vibrational transitions of a solid sphere: Selection rules, Phys. Rev. B 46, 5795 (1992).

[37] M. Montagna, Brillouin and Raman scattering from the acoustic vibrations of spherical particles with a size comparable to the wavelength of the light, Phys. Rev. B 77, 045418 (2008).

[38] L. Quan, Y. Ra'di, D. L. Sounas, and A. Alù, Maximum Willis Coupling in Acoustic Scatterers, Phys. Rev. Lett. 120, 254301 (2018). 